\title{
Coeficientes de similaridade para avaliação da diversidade genética em pinhão-manso por marcadores ISSR
}

\author{
Francisco Linco de Souza TOMAZ¹, Ana Paula Moura da SILVA², Linda Brenna Ribeiro ARAÚJO1, \\ Jonas CUNHA NETO³ ${ }^{3}$ Cândida Hermínia Campos de Magalhães BERTINI ${ }^{4}$
}

\author{
1 Programa de Pós-Graduação em Agronomia/Fitotecnia, Universidade Federal do Ceará, Fortaleza, CE, Brasil. \\ (Orcid: *; 0000-0002-3554-3908) \\ ${ }^{2}$ Embrapa Agrossilvipastoril, Sinop, MT, Brasil. (Orcid: 0000-0001-6073-9243) \\ ${ }^{3}$ Faculdade Centro-Matogrossense, Sorriso, MT, Brasil. (Orcid: 0000-0003-3190-9623) \\ ${ }^{4}$ Centro de Ciências Agrárias, Universidade Federal do Ceará, Fortaleza, CE, Brasil. (Orcid: 0000-0003-2949-5660)
}

*E-mail: lincotomaz@gmail.com (Orcid: 0000-0002-8696-8914)

Recebido em 14/01/2020; Aceito em 16/07/2020; Publicado em 31/07/2020.

RESUMO: Objetivou-se com este trabalho, avaliar a eficiência da utilização de diferentes coeficientes de similaridade na estimação da diversidade genética de Jatropha curcas L. utilizando marcadores moleculares ISSR. O DNA genômico foi extraído a partir de folhas jovens de 43 acessos de pinhão-manso. Matrizes de dissimilaridade genética foram obtidas a partir dos coeficientes Baroni, Coincidência Simples, Hamann, Índice II, Índice III, Jaccard, Nei e Li, Ochiai I, Ochiai II, Rogers e Tanimoto e Sokal e Sneath. Os dendrogramas foram construídos utilizando o método UPGMA e comparados mediante os parâmetros de coeficiente de correlação cofenético, estresse e distorção. Foram estimadas as correlações entre os pares de matrizes pelo teste de Mantel. Houve concordância entre as matrizes originais e as matrizes resultantes do processo de agrupamento para todos os coeficientes estudados. Os índices de Jaccard e Nei e Li não diferiram quanto ao ordenamento dos acessos avaliados e permitiram maior discriminação destes, sendo os mais adequados para avaliar a diversidade genética em pinhão-manso baseada em marcadores moleculares ISSR.

Palavras-chave: dissimilaridade genética; análise de agrupamento; Jatropha curcas L.

\section{Similarity coefficients for evaluation of genetic diversity in jatropha by ISSR markers}

\begin{abstract}
The aim of this work was to evaluate the efficiency of using different similarity coefficients in the estimation of Jatropha curcas L. genetic diversity using ISSR molecular markers. Genomic DNA was extracted from young leaves of the 43 jatropha accessions. Genetic dissimilarity matrices were obtained from the Baroni, Simple Matching, Hamann, Index II, Index III, Jaccard, Nei and Li, Ochiai I, Ochiai II, Rogers and Tanimoto and Sokal and Sneath coefficients. The dendrograms were constructed using the UPGMA method and compared using the co-phenetic correlation coefficient, stress and distortion parameters. Correlations between pairs of matrices were estimated by the Mantel test. There was agreement between the original matrices and the matrices resulting from the grouping process for all the studied coefficients. The Jaccard and Nei and Li indices did not differ in terms of the order of the evaluated accessions and allowed for greater discrimination of these, being the most suitable for assessing genetic diversity in physic nut based on ISSR molecular markers.
\end{abstract}

Keywords: genetic dissimilarity; cluster analysis; Jatropha curcas L.

\section{INTRODUÇÃO}

O pinhão-manso (Jatropha curcas L.) é uma Euphorbiaceae originária da América Central, sendo encontrada espontaneamente em boa parte das regiões intertropicais (FIGUEIREDO et al., 2018). A espécie possui rápido crescimento e baixa exigência hídrica e nutricional, além de apresentar alto teor de óleo nas sementes, o que lhe confere grande potencial para a produção de biodiesel (NÁPOLES et al., 2017; PADILHA et al., 2016). Apesar das características citadas, ainda existem poucos programas de melhoramento para a cultura, quando comparada com outras oleaginosas já estabelecidas como soja, girassol e amendoim (FREITAS et al., 2011).

Tendo em vista que o sucesso de um programa de melhoramento depende principalmente do conhecimento, conservação e utilização da variabilidade genética da espécie, sua determinação torna-se crucial. Atualmente, o uso de marcadores moleculares como referência ao longo dos cromossomos, tem sido a tecnologia básica para o estudo da divergência genética em plantas (BORÉM; CAIXETA, 2016). Dentre os marcadores de DNA mais utilizados, os ISSR (Inter Simple Sequence Repeat) merecem destaque por apresentar facilidade na obtenção dos dados, custo relativamente reduzido e elevados níveis de polimorfismo e reprodutibilidade (CAIXETA et al., 2013). Esta técnica permite, além da caracterização do germoplasma e da construção de mapas genéticos, estimar a similaridade/dissimilaridade genética entre indivíduos (BORÉM; CAIXETA, 2016). 
Para o cálculo das distâncias genéticas entre cultivares ou acessos, diferentes metodologias são encontradas na literatura, tais como: Jaccard; Russel e Rao; Ochiai; Sokal e Sneath; entre outras (CRUZ, 2008). Para o pinhão-manso, a maior parte dos trabalhos utiliza apenas um ou poucos coeficientes, sendo os mais comuns os de Jaccard, Nei e Li e Coincidência Simples (DÍAZ et al., 2017; RIBEIRO et al., 2017; ALKIMIM et al., 2013; OLIVEIRA et al., 2013; SANTOS et al., 2010).

No entanto, considerando que a maioria dos autores não justifica a escolha dos coeficientes de similaridade utilizados e que, a confiabilidade da análise de agrupamento pode ser comprometida pela seleção subjetiva destes, faz-se necessário estudá-los melhor, visando recomendar o coeficiente mais adequado para cada situação (ALBUQUERQUE et al., 2016).

Diante do exposto, objetivou-se com este trabalho, comparar diferentes coeficientes de similaridade para avaliação da diversidade genética em acessos de Jatropha curcas L., utilizando marcadores ISSR.

\section{MATERIAL E MÉTODOS}

Para a realização desta pesquisa, utilizaram-se folhas jovens de 43 acessos de pinhão-manso oriundos da coleção de germoplasma da Universidade Federal do Ceará - UFC (Tabela 1). Após a coleta do material vegetal, realizou-se a extração do DNA genômico de acordo com o protocolo de Doyle; Doyle (1990). A qualidade do DNA extraído foi verificada em gel de agarose $0,8 \%$ e sua quantificação foi realizada em espectrofotômetro NanoDrop 2000 (Thermo Scientific $\left.{ }^{\circledR}\right)$. As amostras de DNA foram armazenadas a $20^{\circ} \mathrm{C}$.

Para as análises moleculares foram utilizados 18 iniciadores ISSR (Integrated DNA Technologies ${ }^{\circledR}$ ) (Tabela 2). As reações de amplificação foram realizadas em termociclador THERM-1000 (Axygen ${ }^{\circledR}$ ), com as seguintes concentrações finais: DNA genômico $(30 \mathrm{ng} / \mu \mathrm{L})$; tampão (1X); $\mathrm{MgCl}(2 \mathrm{mM})$; dNTPs $(0,2 \mathrm{mM})$; iniciador $(0,8 \mu \mathrm{M}) \mathrm{e}$ Taq DNA polimerase (1 U) (Go'Taq Flexi DNA Polymerase, Promega $\left.{ }^{\circledR}\right)$. As reações consistiram de uma desnaturação inicial a $94^{\circ} \mathrm{C}$ por 5 minutos, seguida por 40 ciclos nas seguintes condições: desnaturação a $94^{\circ} \mathrm{C}$ por 1 minuto, anelamento a 50,53 ou $55^{\circ} \mathrm{C}$, a depender do iniciador, por 30 segundos, e extensão por 1 minuto a $72^{\circ} \mathrm{C}$, além de uma extensão final a $72^{\circ} \mathrm{C}$ por 10 minutos. Os produtos resultantes das amplificações foram separados por eletroforese horizontal utilizando-se gel de agarose 1,2\%, imerso em tampão TBE $0,5 \mathrm{X}$, a 80 volts durante 3 horas. Foram utilizados marcadores de 100 pb (Invitrogen ${ }^{\circledR}$ ) para comparar o tamanho dos fragmentos amplificados.

Os géis foram corados com brometo de etídio (10 $\mathrm{mg} / \mathrm{mL}$ ) e fotografados em fotodocumentador Gel Logic 212 Pro (Carestream $\AA$ ). A partir da análise dos padrões formados, procedeu-se à montagem da matriz binária, com atribuição de escores baseados na presença (1) e na ausência (0) das 208 bandas geradas. Onze coeficientes de similaridade foram analisados (Tabela 3). As estimativas de similaridade $\left(\mathrm{sg}_{\mathrm{ij}}\right)$ entre os genótipos foram convertidas em dissimilaridade $\left(\mathrm{dg}_{\mathrm{ii}}\right)$ por meio do complemento aritmético dos coeficientes utilizados, obtidos pela expressão: $\mathrm{dg}_{\mathrm{ij}}=1-\mathrm{sg}_{\mathrm{ij}}$ (CRUZ et al., 2012).
Tabela 1. Identificação e origem dos acessos de pinhão-manso provenientes da coleção de germoplasma da UFC.

Table 1. Identification and origin of jatropha accessions from the UFC germplasm collection.

\begin{tabular}{|c|c|c|}
\hline Identificação & Acesso & Origem \\
\hline 1 & FSV-PI (2) & Piauí \\
\hline 2 & FSV-PI (3) & Piauí \\
\hline 3 & FB-T 4 (1) & Ceará \\
\hline 4 & FB-T 4 (2) & Ceará \\
\hline 5 & FB-T 4 (3) & Ceará \\
\hline 6 & FB-T 4 (4) & Ceará \\
\hline 7 & FB-T 4 (5) & Ceará \\
\hline 8 & FB-T 5 & Ceará \\
\hline 9 & RV P 2 & Ceará \\
\hline 10 & ASS. FACUNDO & Ceará \\
\hline 11 & G-P 1 (1) & Ceará \\
\hline 12 & G-P 1 (2) & Ceará \\
\hline 13 & G-P 1 (3) & Ceará \\
\hline 14 & G-P 1 (4) & Ceará \\
\hline 15 & G-P 1 (5) & Ceará \\
\hline 16 & G-P 1 (6) & Ceará \\
\hline 17 & G-P 3 & Ceará \\
\hline 18 & BOA NOVA & Ceará \\
\hline 19 & FLS-L 1 (1) & Ceará \\
\hline 20 & FLS-L 1 (2) & Ceará \\
\hline 21 & FLS-L 5 (3) & Ceará \\
\hline 22 & FLS-L 6 (4) & Ceará \\
\hline 23 & FLS-L 7 (5) & Ceará \\
\hline 24 & CNPA II-P29 & Paraíba \\
\hline 25 & CNPA III-P2 & Paraíba \\
\hline 26 & CNPA VII-P2 & Paraíba \\
\hline 27 & CNPA VIII-P6 & Paraíba \\
\hline 28 & CNPA XI-P1 & Paraíba \\
\hline 29 & CNPA XII-P7 & Paraíba \\
\hline 30 & CNPA XIV-P1 & Paraíba \\
\hline 31 & CNPA XV-P5 & Paraíba \\
\hline 32 & CNPA XVI-P12 & Paraíba \\
\hline 33 & UFRRJ FMI002 & Rio de Janeiro \\
\hline 34 & UFRRJ FMI346 & Rio de Janeiro \\
\hline 35 & UFRRJ FMI355 & Rio de Janeiro \\
\hline 36 & UFRRJ FMI383 & Rio de Janeiro \\
\hline 37 & UFRRJ FMI858 & Rio de Janeiro \\
\hline 38 & UFRRJ FMI860 & Rio de Janeiro \\
\hline 39 & UFRRJ FMI869 & Rio de Janeiro \\
\hline 40 & UFRRJ FMI872 & Rio de Janeiro \\
\hline 41 & UFRRJ FMI874 & Rio de Janeiro \\
\hline 42 & UFRRJ FMI875 & Rio de Janeiro \\
\hline 43 & NF-RN & Rio Grande do Norte \\
\hline
\end{tabular}

Tabela 2. Iniciadores ISSR e sequências 5'-3'. Table 2. ISSR primers and 5'-3' sequences.

\begin{tabular}{|c|c|}
\hline Iniciador & Sequência 5'-3' \\
\hline UBC 807 & $(\mathrm{AG})_{8} \mathrm{~T}$ \\
\hline UBC 808 & $(\mathrm{AG})_{8} \mathrm{C}$ \\
\hline UBC 809 & $(\mathrm{AG}){ }_{8} \mathrm{G}$ \\
\hline UBC 810 & $(\mathrm{CA}){ }_{8} \mathrm{~T}$ \\
\hline UBC 811 & $(\mathrm{GA}){ }_{8} \mathrm{C}$ \\
\hline UBC 825 & (AC) $8 \mathrm{~T}$ \\
\hline UBC 827 & $(\mathrm{CA})_{8} \mathrm{G}$ \\
\hline UBC 828 & $(\mathrm{TG}) 8 \mathrm{~A}$ \\
\hline UBC 862 & $(\mathrm{AGC})_{6}$ \\
\hline UBC 873 & $(\mathrm{GACA})_{4}$ \\
\hline UBC 880 & $(\mathrm{GGAGA})_{3}$ \\
\hline I810 & $(\mathrm{GA})_{8} \mathrm{~T}$ \\
\hline I825 & (AC) $7 \mathrm{AT}$ \\
\hline I826 & $(\mathrm{AC}){ }_{8} \mathrm{C}$ \\
\hline I827 & $(\mathrm{AC}){ }_{8} \mathrm{G}$ \\
\hline I834 & (AG)8YT \\
\hline I842 & $(\mathrm{GA})_{8} \mathrm{YG}$ \\
\hline I888 & $\mathrm{BDB}(\mathrm{CA})_{7}$ \\
\hline
\end{tabular}


Tabela 3. Coeficientes de similaridade utilizados para obtenção da matriz de dissimilaridade genética entre 43 acessos de pinhão-manso com base em marcadores moleculares ISSR.

Table 3. Similarity coefficients used to get the genetic dissimilarity matrix among 43 jatropha accessions based on ISSR molecular markers.

\begin{tabular}{cc}
\hline Coeficiente & Expressão da similaridade $(1)^{(1)}$ \\
\hline Baroni & {$[\mathrm{a}+\sqrt{(\mathrm{ad})}] /[\mathrm{a}+\mathrm{b}+\mathrm{c}+\sqrt{(\mathrm{ad})}]$} \\
Coincidência Simples & $(\mathrm{a}+\mathrm{d}) /(\mathrm{a}+\mathrm{b}+\mathrm{c}+\mathrm{d})$ \\
Hamann & {$[(\mathrm{a}+\mathrm{d})-(\mathrm{b}+\mathrm{c})] /(\mathrm{a}+\mathrm{b}+\mathrm{c}+\mathrm{d})$} \\
Índice II & $0,5[\mathrm{a} /(\mathrm{a}+\mathrm{b})+\mathrm{a} /(\mathrm{a}+\mathrm{c})]$ \\
Índice III & $0,25\left[\begin{array}{l}\mathrm{a} /(\mathrm{a}+\mathrm{b})+\mathrm{a} /(\mathrm{a}+\mathrm{c})+] \\
\mathrm{d} /(\mathrm{d}+\mathrm{b})+\mathrm{d} /(\mathrm{d}+\mathrm{c})\end{array}\right]$ \\
Jaccard & $\mathrm{a} /(\mathrm{a}+\mathrm{b}+\mathrm{c})$ \\
Nei e Li & $2 \mathrm{a} /(2 \mathrm{a}+\mathrm{b}+\mathrm{c})$ \\
Ochiai I & $\mathrm{a} / \sqrt{(\mathrm{a}+\mathrm{b})(\mathrm{a}+\mathrm{c})}$ \\
Ochiai II & $\mathrm{ad} / \sqrt{(\mathrm{a}+\mathrm{b})(\mathrm{a}+\mathrm{c})(\mathrm{b}+\mathrm{d})(\mathrm{c}+\mathrm{d})}$ \\
Rogers e Tanimoto & $(\mathrm{a}+\mathrm{d}) /[\mathrm{a}+2(\mathrm{~b}+\mathrm{c})+\mathrm{d}]$ \\
Sokal e Sneath & $2(\mathrm{a}+\mathrm{d}) /[2(\mathrm{a}+\mathrm{d})+\mathrm{b}+\mathrm{c}]$ \\
\hline (1) $\mathrm{a}=1: 1 ; \mathrm{b}=1: 0 ; \mathrm{c}=0: 1 ; \mathrm{d}=0: 0$. Fonte: Adaptado de Cruz $(2008)$.
\end{tabular}

A partir das matrizes de dissimilaridade geradas por cada índice, foram construídos dendrogramas mediante o método de agrupamento hierárquico da ligação média entre grupos UPGMA (Unweighted Pair Group Method with Arithmetic Mean), e a validação da representação gráfica foi determinada pelo coeficiente de correlação cofenético (CCC) (SOKAL; ROHLF, 1962), estresse e distorção (KRUSKAL, 1962). O ponto de corte estabelecido foi a distância genética média entre os pares de indivíduos, obtida para cada índice. Para as estimativas de correlações entre todos os pares de matrizes obtidas, realizou-se o teste de Mantel com 1.000 permutações (MANTEL, 1967).

Os coeficientes de similaridade foram comparados quanto à eficiência de projeção das distâncias originais no espaço bidimensional mediante os parâmetros estresse $(\mathrm{S})$ e distorção $(1-\alpha)$, expressos pelas equações 1 e 2 :

$$
\mathrm{S}=\sqrt{\sum_{\mathrm{i}<\mathrm{j}}\left(\mathrm{d}_{\mathrm{oij}}-\mathrm{d}_{\mathrm{gij}}\right)^{2} / \sum_{\mathrm{i}<\mathrm{j}} \mathrm{d}_{\mathrm{oij}}}
$$

em que: $\mathrm{dg}_{\mathrm{ij}}$ e do $\mathrm{o}_{\mathrm{ij}}$ são as distâncias gráficas (espaço bidimensional) e originais (espaço $\mathrm{n}$ dimensional), respectivamente, de todos os pares de genótipos i e j.

$$
\alpha=\sum_{\mathrm{i}<\mathrm{j}} \mathrm{d}_{\mathrm{gij}} / \sum_{\mathrm{i}<\mathrm{j}} \mathrm{d}_{\mathrm{oij}}
$$

Esta representação estatística de estresse (soma de quadrados de resíduos padronizados), proposta por Kruskal (1964), é um parâmetro que determina a qualidade do ajuste da projeção gráfica.

As análises estatísticas foram realizadas utilizando o programa computacional GENES (CRUZ, 2013) e os dendrogramas foram obtidos pelo software $\mathrm{R}$ ( $\mathrm{R}$ CORE TEAM, 2019).

\section{RESULTADOS}

A quantidade de grupos formados pelo método de agrupamento UPGMA apresentou baixa variação, com amplitude de 9 a 12 grupos (Figuras 1 e 2). Dentre os coeficientes avaliados, Jaccard, Nei e Li e Ochiai I, apresentaram a maior capacidade discriminatória, haja vista terem formado o maior número de grupos (12). Por outro lado, o agrupamento obtido a partir do coeficiente Índice II, gerou apenas 9 grupos, evidenciando baixa eficiência deste em discriminar os acessos estudados.

Os valores de correlação entre as matrizes de dissimilaridade obtidos mediante a maioria dos coeficientes avaliados foram elevados (acima de 0,90), evidenciando uma forte associação entre eles (Tabela 4). Entretanto, o coeficiente de Hamann apresentou valores de correlação inferiores aos demais, principalmente em relação a Rogers e Tanimoto $(0,22)$, Sokal e Sneath $(0,22)$, Índice III $(0,29)$ e Ochiai II $(0,30)$.

Os dez pares de acessos mais similares, bem como os dez pares mais divergentes, são os mesmos obtidos com os coeficientes de Jaccard e Nei e Li. No entanto, há diferença quanto ao ordenamento dos genótipos mais dissimilares quando o índice de Ochiai I é utilizado (Tabela 5).

Para verificar a capacidade dos dendrogramas em reproduzir as matrizes de dissimilaridade, calculou-se o CCC (Tabela 6).

Tabela 4. Correlações entre matrizes de dissimilaridade obtidas a partir da utilização dos onze coeficientes de similaridade avaliados.

\begin{tabular}{|c|c|c|c|c|c|c|c|c|c|c|}
\hline & B & $\mathrm{C}$ & $\mathrm{D}$ & $E$ & $\mathrm{~F}$ & $G$ & $\mathrm{H}$ & I & $J$ & $\mathrm{~K}$ \\
\hline $\mathrm{A}$ & 0,98 & 0,98 & 0,90 & 0,99 & 0,98 & 0,98 & 0,91 & 0,99 & 0,99 & 0,99 \\
\hline B & & 0,99 & 0,90 & 0,99 & 0,92 & 0,92 & 0,91 & 0,99 & 0,99 & 0,99 \\
\hline $\mathrm{C}$ & & & 0,59 & 0,29 & 0,92 & 0,92 & 0,59 & 0,30 & 0,22 & 0,22 \\
\hline $\mathrm{D}$ & & & & 0,94 & 0,99 & 0,99 & 0,99 & 0,94 & 0,91 & 0,90 \\
\hline $\mathrm{E}$ & & & & & 0,94 & 0,94 & 0,94 & 0,99 & 0,99 & 0,99 \\
\hline $\mathrm{F}$ & & & & & & 0,99 & 0,99 & 0,95 & 0,92 & 0,91 \\
\hline G & & & & & & & 0,99 & 0,95 & 0,92 & 0,91 \\
\hline $\mathrm{H}$ & & & & & & & & 0,95 & 0,91 & 0,91 \\
\hline $\mathrm{I}$ & & & & & & & & & 0,99 & 0,99 \\
\hline $\mathrm{J}$ & & & & & & & & & & 0,99 \\
\hline
\end{tabular}
Table 4. Correlations between dissimilarity matrices obtained from the use of the eleven similarity coefficients evaluated.

Correlações significativas a 1\% de probabilidade pelo teste de Mantel, baseado em 1000 simulações. Baroni (A); Coincidência Simples (B); Hamann (C); Índice II (D); Indice III (E); Jaccard (F); Nei e Li (G); Ochiai I (H); Ochiai II (I); Rogers e Tanimoto (J); Sokal e Sneath (K). 
Tomaz et al.

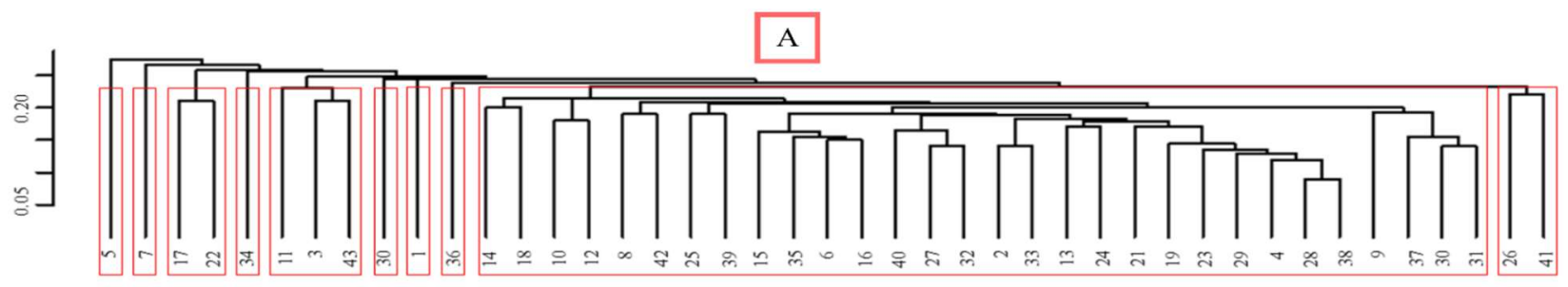

B

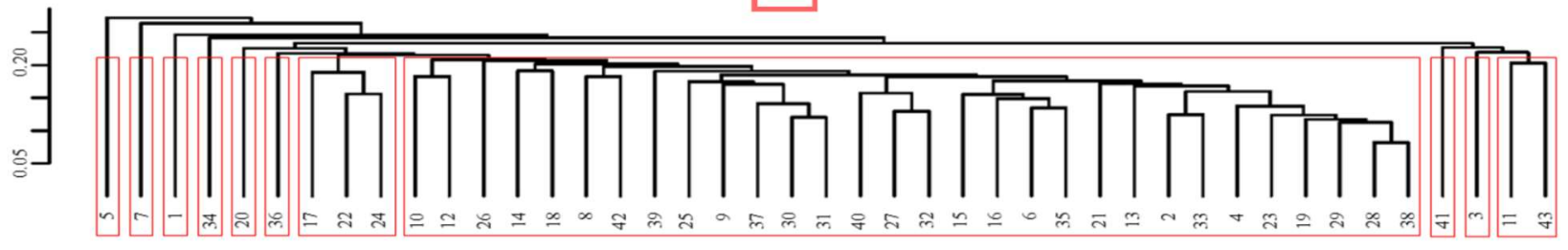

$\mathrm{C}$

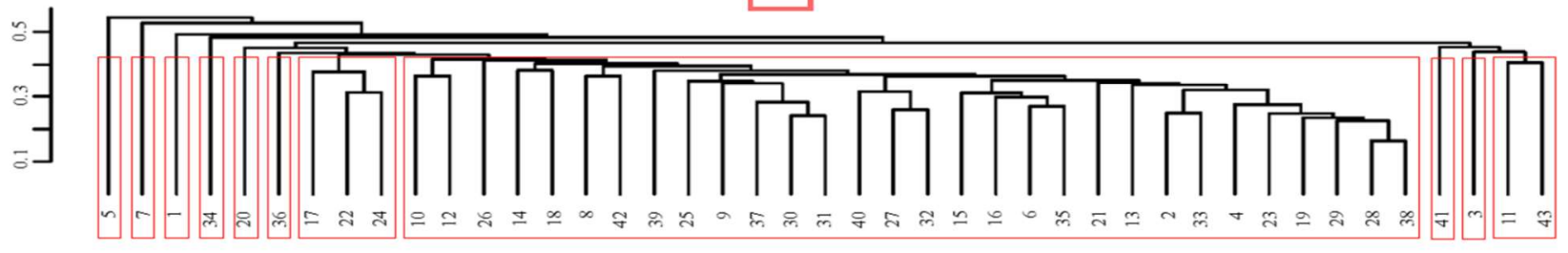

D

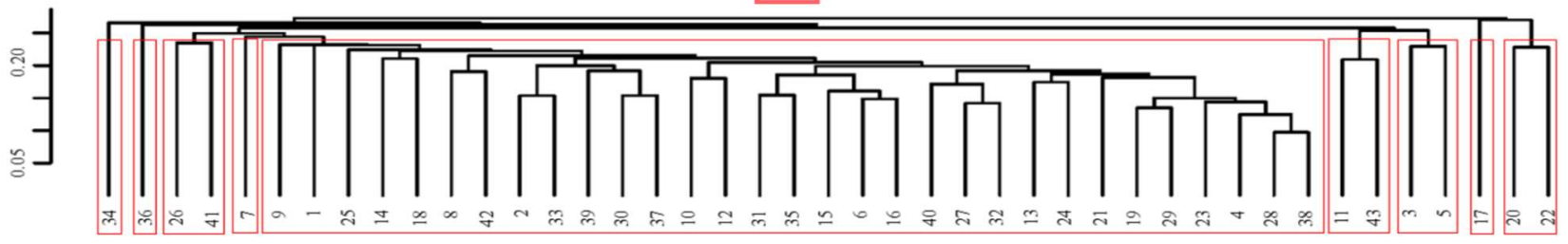

E

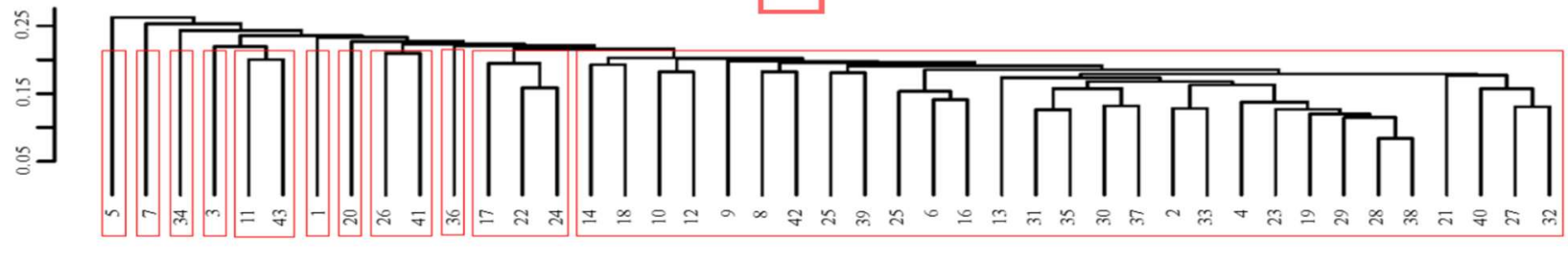

$\mathrm{F}$

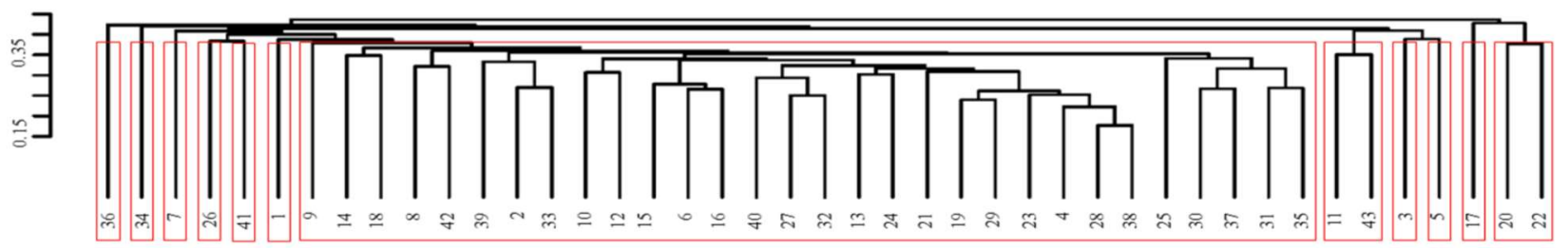

Figura 1. Dendrogramas representativos da dissimilaridade genética entre 43 acessos de pinhão-manso, construídos a partir das matrizes de distâncias genéticas obtidas pelo complemento dos coeficientes de similaridade Baroni (A), Coincidência Simples (B), Hamann (C), Índice II (D), Índice III (E) e Jaccard (F).

Figure 1. Dendrograms representing genetic dissimilarity among 43 accessions of jatropha, constructed from the genetic distance matrices obtained by complementing the similarity coefficients Baroni (A), Simple Matching (B), Hamann (C), Index II (D), Index III (E) and Jaccard $(\mathrm{F})$. 
G

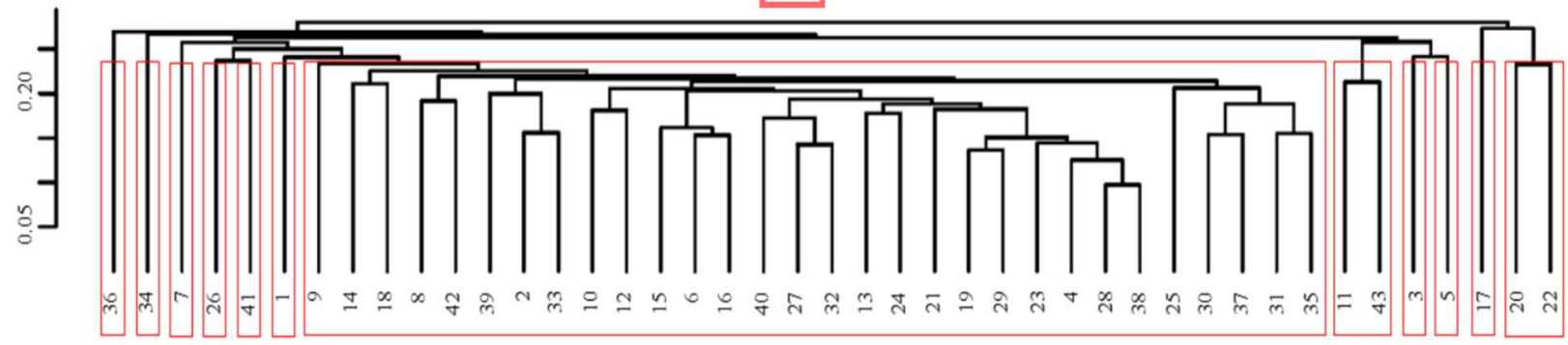

$\mathrm{H}$
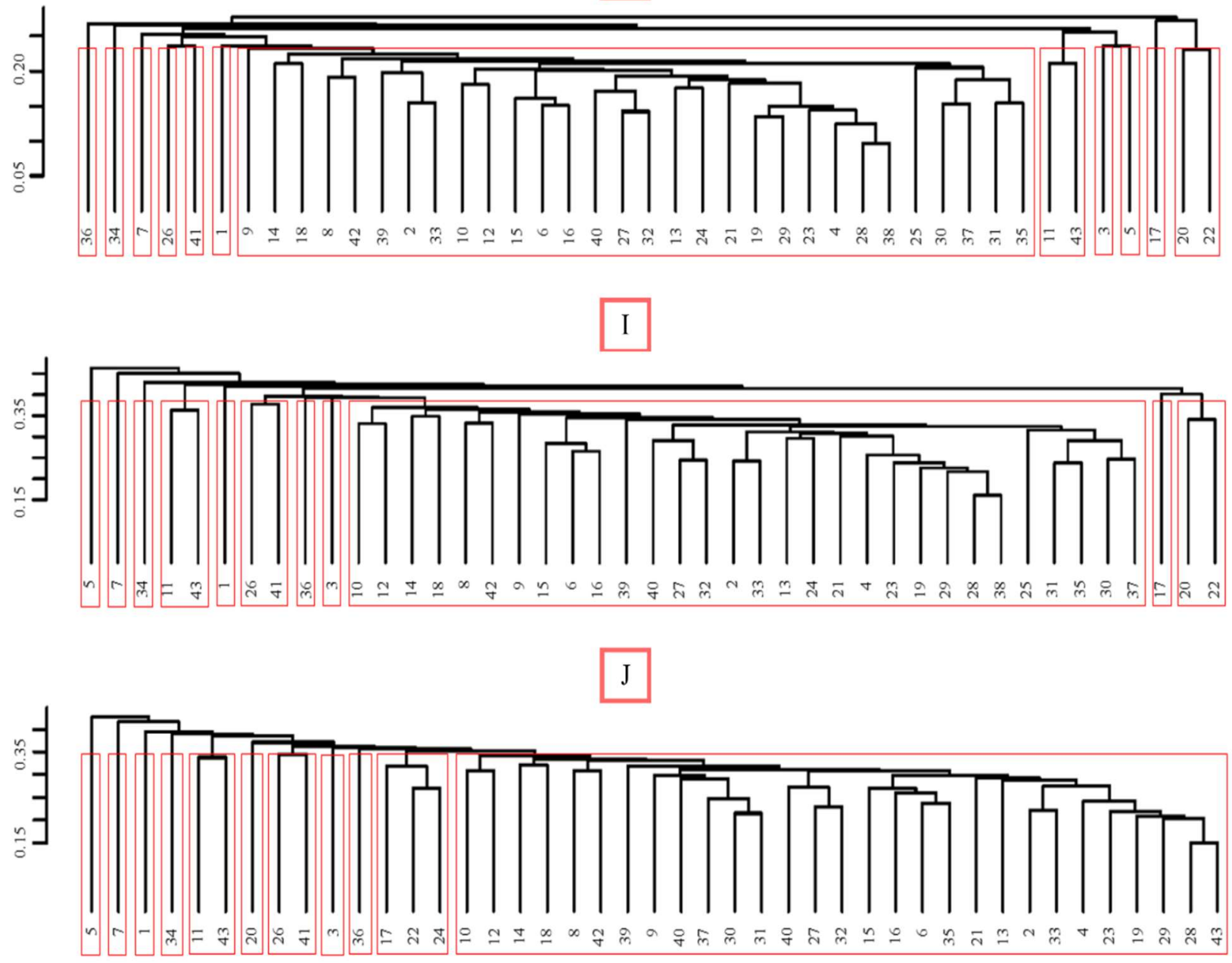

K

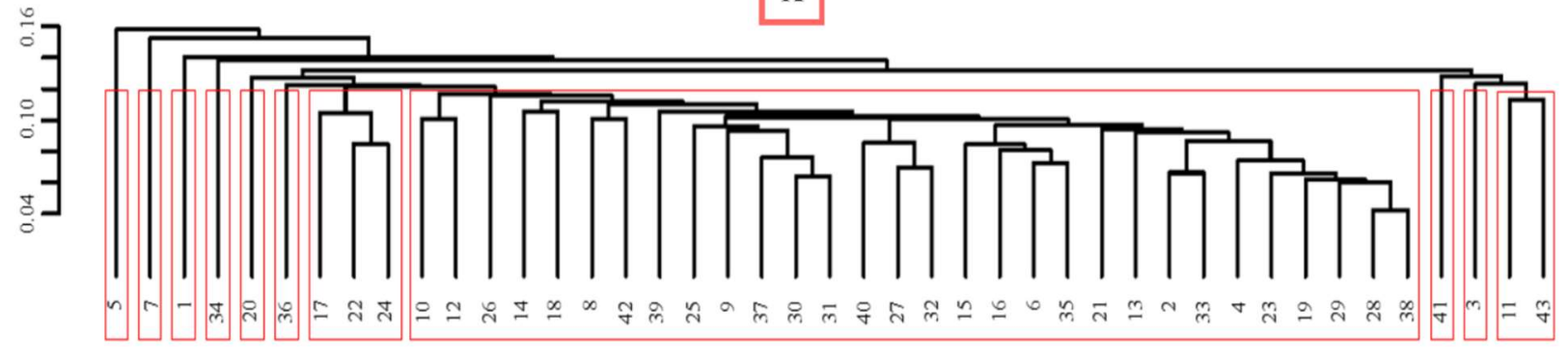

Figura 2. Dendrogramas representativos da dissimilaridade genética entre 43 acessos de pinhão-manso, construídos a partir das matrizes de distâncias genéticas obtidas pelo complemento dos coeficientes de similaridade Nei e Li (G), Ochiai I (H), Ochiai II (I), Rogers e Tanimoto (J) e Sokal e Sneath (K).

Figure 2. Dendrograms representing genetic dissimilarity among 43 accessions of jatropha, constructed from the genetic distance matrices obtained by complementing the similarity coefficients Nei e Li (G), Ochiai I (H), Ochiai II (I), Rogers e Tanimoto (J) e Sokal e Sneath (K). 
Tabela 5. Acessos de pinhão-manso com maior e menor similaridade e suas respectivas distâncias genéticas.

Table 5. Jatropha accessions with greater and less similarity and their respective genetic distances.

\begin{tabular}{|c|c|c|c|}
\hline & Jaccard & Nei e Li & Ochiai I \\
\hline \multirow{10}{*}{ 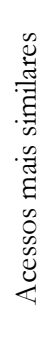 } & 28 e $38(0,177)$ & 28 e $38(0,097)$ & 28 e $38(0,097)$ \\
\hline & 32 e $38(0,200)$ & 32 e $38(0,111)$ & 32 e $38(0,111)$ \\
\hline & 29 e $38(0,218)$ & 29 e $38(0,122)$ & 29 e $38(0,122)$ \\
\hline & 35 e $38(0,219)$ & 35 e $38(0,123)$ & 35 e $38(0,122)$ \\
\hline & 4 e $28(0,222)$ & 4 e $28(0,125)$ & 4 e $28(0,125)$ \\
\hline & 4 e $38(0,222)$ & 4 e $38(0,125)$ & 4 e $38(0,125)$ \\
\hline & 23 e $38(0,224)$ & 23 e $38(0,126)$ & 23 e $38(0,126)$ \\
\hline & 19 e $38(0,227)$ & 19 e $38(0,128)$ & 19 e $38(0,128)$ \\
\hline & 19 e $29(0,240)$ & 19 e $29(0,136)$ & 19 e $29(0,135)$ \\
\hline & 28 e $29(0,245)$ & 28 e $29(0,140)$ & 28 e $29(0,139)$ \\
\hline \multirow{10}{*}{ 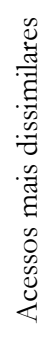 } & 5 e $22(0,534)$ & 5 e $22(0,364)$ & 5 e $22(0,349)$ \\
\hline & 7 e $17(0,519)$ & 7 e $17(0,350)$ & 3 e $22(0,344)$ \\
\hline & 5 e $36(0,515)$ & 5 e $36(0,347)$ & 7 e $17(0,342)$ \\
\hline & 3 e $22(0,515)$ & 3 e $22(0,347)$ & 6 e $20(0,340)$ \\
\hline & 5 e $31(0,512)$ & 5 e $31(0,344)$ & 5 e $36(0,338)$ \\
\hline & 6 e $20(0,509)$ & 6 e $20(0,341)$ & 1 e $26(0,333)$ \\
\hline & 5 e $20(0,504)$ & 5 e $20(0,337)$ & 11 e $36(0,333)$ \\
\hline & 1 e $26(0,504)$ & 1 e $26(0,337)$ & 3 e $20(0,332)$ \\
\hline & 3 e $20(0,500)$ & 3 e $20(0,333)$ & 5 e $31(0,332)$ \\
\hline & 5 e $30(0,500)$ & 5 e $30(0,333)$ & 9 e $11(0,329)$ \\
\hline
\end{tabular}

Tabela 6. Coeficiente de correlação cofenético (CCC), estresse e distorção entre a matriz original de distâncias e a matriz resultante do processo de agrupamento.

Table 6. Cofenetic correlation coefficient (CCC), stress and distortion between the original distance matrix and the resulting matrix of the clustering process.

\begin{tabular}{cccc}
\hline Coeficiente & CCC & Estresse (\%) & Distorção $(\%)$ \\
\hline A & 0,76 & 63,11 & 59,59 \\
B & 0,77 & 65,59 & 62,66 \\
C & 0,77 & 65,59 & 62,66 \\
D & 0,77 & 67,95 & 65,29 \\
E & 0,76 & 69,49 & 66,90 \\
F & 0,77 & 71,50 & 69,19 \\
G & 0,76 & 66,95 & 64,31 \\
H & 0,77 & 67,75 & 65,12 \\
I & 0,79 & 70,47 & 68,44 \\
J & 0,77 & 69,93 & 67,40 \\
K & 0,76 & 63,11 & 59,59 \\
\hline
\end{tabular}

Baroni (A); Coincidência Simples (B); Hamann (C); Índice II (D); Índice III (E); Jaccard (F); Nei e Li (G); Ochiai I (H); Ochiai II (I); Rogers e Tanimoto (J); Sokal e Sneath (K).

\section{DISCUSSÃO}

Com base na análise dos dendrogramas, houve concordância entre o agrupamento realizado pelos coeficientes de Jaccard, Nei e Li e Ochiai I, que diferiram dos grupos formados pelos demais índices (Figuras 1 e 2). Os coeficientes citados apresentam propriedades semelhantes devido ao fato de não considerarem a ausência conjunta (0:0), indicada pela letra d nas expressões de similaridade apresentadas na Tabela 3 (MEYER et al., 2004). Devido às propriedades bioquímicas dos marcadores moleculares dominantes, como ISSR, Cruz et al. (2012) sugerem a utilização de coeficientes que desconsideram a ausência conjunta para estudos de diversidade genética em espécies vegetais, o que pode ser reforçado pela melhor discriminação dos agrupamentos destes índices, mostrada no presente estudo.

Os grupos formados não apresentaram relação com a origem dos acessos para nenhum dos coeficientes testados, não sendo capazes de discriminá-los pelos locais de coleta. No entanto, os acessos de pinhão-manso do Brasil podem ter ascendentes comuns, o que dificulta esta separação (ROSADO et al., 2010).

A baixa correlação envolvendo o índice de Hamann pode ser devido ao seu intervalo de ocorrência $(-1,1)$, uma vez que todos os demais coeficientes estudados variam no intervalo entre 0 e 1 . Vale ressaltar que a reduzida correlação entre o índice supracitado e os índices de Rogers e Tanimoto, Sokal e Sneath, Índice III e Ochiai II (Tabela 4), pode resultar em divergências no agrupamento e/ou no ordenamento dos genótipos, gerando dificuldades na interpretação dos resultados.

O conhecimento dos acessos mais divergentes orienta o melhorista durante o processo de seleção de parentais em programas de melhoramento genético. Portanto, é importante que o ordenamento destes acessos seja mantido, uma vez que alterações nos grupos podem modificar a interpretação da relação existente entre eles (BALESTRE et al., 2008). Neste sentido, os coeficientes de Jaccard e Nei e Li mantiveram o ordenamento dos acessos avaliados e os agruparam de forma similar, sendo considerados os mais adequados ao uso em programas de melhoramento (Tabela 5).

De acordo com Sokal e Rohlf (1962), valores de CCC inferiores a 0,7 indicam a inadequação do método de agrupamento para resumir a informação do conjunto de dados. Constatou-se, portanto, a adequação entre as matrizes originais e as matrizes resultantes do processo de agrupamento, uma vez que todos os coeficientes apresentaram valores de CCC superiores a 0,76 (Tabela 6).

A projeção das distâncias genéticas dos coeficientes de similaridade, em um espaço bidimensional, considerando dois critérios (estresse e distorção entre as distâncias originais e estimadas), apresentou baixa variação (Tabela 6), o que também foi observado por Balestre et al. (2008) ao estudar o milho. Os menores valores de estresse e distorção foram verificados para os coeficientes de Baroni e Sokal e Sneath.

Segundo Kruskal (1964), o estresse é um parâmetro que determina a qualidade do ajuste da projeção gráfica, sendo classificado conforme os níveis de 40\%, 20\%, 10\%, 5\% e 0\% de estresse, os quais indicam que o ajuste será, respectivamente, insatisfatório, regular, bom, excelente e perfeito. Portanto, os valores de estresse foram considerados insatisfatórios para todos os coeficientes avaliados (Tabela 6), sugerindo que o método da projeção bidimensional não foi adequado para representar as matrizes de similaridade. Resultado semelhante foi verificado por Dencic et al. (2016) que, comparando coeficientes de similaridade na cultura do trigo, por meio de marcadores moleculares dominantes, concluíram que todos os coeficientes avaliados apresentaram nível de estresse inadequado.

Relacionando as expressões de similaridade com a estrutura dos agrupamentos, verificou-se elevada semelhança entre os índices que possuem propriedades comuns. Os coeficientes Índice II, Jaccard, Nei e Li e Ochiai I, desconsideram a ausência conjunta de bandas. Ao passo que os índices Baroni, Coincidência Simples, Hamann, Índice III, Ochiai II, Rogers e Tanimoto e Sokal e Sneath, consideram tal ausência em suas expressões. Estes resultados corroboram com os encontrados por Meyer et al. (2004) que, avaliando a influência de diferentes coeficientes de similaridade sobre a 
análise de agrupamento, utilizando marcadores AFLP e RAPD, também verificaram correspondência entre o agrupamento proveniente de índices que compartilham as mesmas propriedades.

Em estudos genéticos com marcadores moleculares dominantes (ISSR), as concordâncias do tipo 0 e 0 , em casos particulares, não significam reais similaridades genéticas. Assim sendo, coeficientes que excluem a co-ocorrência negativa das expressões de similaridade tornam-se mais adequados para utilização com marcadores deste tipo (CRUZ et al., 2012). Além disso, os coeficientes com maior capacidade discriminatória (Jaccard e Nei e Li) estão entre os que apresentaram as maiores correlações $(0,99)$, indicando que a utilização de qualquer um destes índices produzirá resultados similares.

\section{CONCLUSÕES}

Os coeficientes de similaridade de Jaccard e Nei e Li não diferiram quanto ao ordenamento dos acessos avaliados e permitiram maior discriminação destes, sendo considerados os mais adequados para avaliação da diversidade genética de pinhão-manso com base em marcadores moleculares ISSR.

\section{AGRADECIMENTOS}

À Petrobras (Projeto PetroBio Girassol, subprojeto IV: Avaliação de recursos genéticos de Jatropha curcas L.) pelo apoio financeiro.

\section{REFERÊNCIAS}

ALBUQUERQUE, M. A.; SILVA, E. L.; BARROS, K. N. N. O.; XAVIER JUNIOR, S. F. A. Comparação entre coeficientes de similaridade: uma aplicação em ciências florestais. Matemática e Estatística em Foco, Uberlândia, v. 4, n. 2, p. 102-114, 2016.

ALKIMIM, E. R.; SOUSA, T. V.; SOARES, B. O.; SOUZA, D. A.; JUHÁSZ, A. C. P.; NIETSCHE, S.; COSTA, M. R. Genetic diversity and molecular characterization of physic nut genotypes from the active germplasm bank of the Agricultural Research Company of Minas Gerais, Brazil. African Journal of Biotechnology, Nairobi, v. 12, n. 9, p. 907-913, mar. 2013.

BALESTRE, M.; VON PINHO, R. G.; SOUZA, J. C.; LIMA, J. L. Comparison of maize similarity and dissimilarity genetic coefficients based on microsatellite markers. Genetics and Molecular Research, Ribeirão Preto, v. 7, n. 3, p. 695-705, 2008. DOI: http://dx.doi.org/10.4238/vol7-3gmr458

BORÉM, A.; CAIXETA, E. T. Marcadores moleculares. 3. ed. Viçosa: UFV, 2016. 385 p.

CAIXETA, E. T.; FERRÃO, L. F. V.; MACIELZAMBOLIM, E. Marcadores moleculares. In: BORÉM, A.; FRITSCHE-NETO, R. (Ed.). Biotecnologia aplicada ao melhoramento de plantas. Viçosa: UFV, 2013. p. 25-42.

CRUZ, C. D. GENES - a software package for analysis in experimental statistics and quantitative genetics. Acta Scientiarum, Maringá, v. 35, n. 3, p. 271-276, 2013. DOI: http://dx.doi.org/10.4025/actasciagron.v35i3.21251

CRUZ, C. D. Programa GENES - diversidade genética. 1. ed. Viçosa: UFV, 2008. 278 p.

CRUZ, C. D.; REGAZZI, A. J.; CARNEIRO, P. C. S. Modelos biométricos aplicados ao melhoramento genético. 4. ed. Viçosa: UFV, 2012. 514 p.

DENCIC, S.; DEPAUW, R.; MOMCILOVIC, V.; ACIN, V. Comparison of similarity coefficients used for cluster analysis based on SSR markers in sister line wheat cultivars. Genetika, Zemun, v. 48, n. 1, p. 219-232, 2016. DOI: https://dx.doi.org/10.2298/GENSR1601219D

DÍAZ, B. G.; ARGOLLO, D. M.; FRANCO, M. C.; NUCCI, S. M.; SIQUEIRA, W. J.; DE LAAT, D. M.; COLOMBO, C. A. High genetic diversity of Jatropha curcas L. assessed by ISSR. Genetics and Molecular Research, Ribeirão Preto, v. 16, n. 2, p. 1-12, 2017. DOI: http:/ /dx.doi.org/10.4238/gmr16029683

DOYLE, J. J.; DOYLE, J. L. Isolation of plant DNA from fresh tissue. Focus, Atlanta, v. 12, n. 1, p. 13-15, 1990.

FIGUEIREDO, N. P.; CORRÊA, A. A. P.; MORAES, A. C. P.; VIANNA, V. F.; DI MAURO, A. O.; UNÊDATREVISOLI, S. H. Influence of supplementation of the culture medium on in vitro development of Jatropha curcas L. Científica, Jaboticabal, v. 46, n.4, p. 398-402, 2018. DOI: 5529.2018v46n4p398-402 http://dx.doi.org/10.15361/1984-

FREITAS, R. G.; MISSIO, R. F.; MATOS, F. S.; RESENDE, M. D. V.; DIAS, L. A. S. Genetic evaluation of Jatropha curcas L.: an important oilseed for biodiesel production. Genetics and Molecular Research, Ribeirão Preto, v. 10, n. 3, p. 1490-1498, 2011. DOI: http:/ /dx.doi.org/10.4238/vol10-3gmr1146

KRUSKAL, J. B. Multidimensional scaling by optmizing goodness of fit to a nometric hypothesis. Psychometrika, Williamsburg, v. 29, n. 1, p. 1-27, 1964. DOI: https://dx.doi.org/10.1007/BF02289565

MANTEL, N. The detection of disease clustering and a generalized regression approach. Cancer Research, Philadelphia, v. 27, n. 2, p. 209-220, 1967.

MEYER, A. D. S.; GARCIA, A. A. F.; SOUZA, A. P. D.; SOUZA JR., C. L. D. Comparison of similarity coefficients used for cluster analysis with dominant markers in maize (Zea mays L.). Genetics and Molecular Biology, Ribeirão Preto, v. 27, n. 1, p. 83-91, 2004. DOI: https://dx.doi.org/10.1590/S1415-47572004000100014

NÁPOLES, F. A. M.; SOUZA, J. T. A.; OLIVEIRA, S. J. C.; MONTENEGRO, F. T.; AZEVEDO, C. A. V. Utilização de manipueira e urina de vaca como fonte de adubação para a cultura do pinhão-manso (Jatropha curcas L.). Agronegócio e Meio Ambiente, Maringá, v. 10, n. 1, p. 83-102, $2017 . \quad$ DOI: http://dx.doi.org/10.17765/2176-9168.2017v10n1p83102

OLIVEIRA, V. D.; RABBANI, A. R. C.; SILVA, A. V. C. D.; LÉDO, A. D. S. Genetic variability in physic nuts cultivated in Northeastern Brazil. Ciência Rural, Santa Maria, v. 43, n. 6, p. 978-984, 2013. DOI: http://dx.doi.org/10.1590/S0103-84782013005000060

PADILHA, N. S.; SILVA, C. J.; PEREIRA, S. B.; SILVA, J. A. N.; HEID, D. M.; BOTTEGA, S. P.; SCALON, S. P. Q. Crescimento inicial do pinhão-manso submetido a diferentes regimes hídricos em latossolo vermelho distrófico. Ciência Florestal, Santa Maria, v. 26, n. 2, p. 513-521, 2016.2 DOI: http://dx.doi.org/10.5902/1980509822752

R CORE TEAM. R: A language and environment for statistical computing. R Foundation for Statistical Computing, Vienna, 2019. Available on $<$ https://www.R-project.org/>. 
RIBEIRO, D. O.; SILVA-MANN, R.; CARVALHO, S. V. Á.; SOUZA, E. M. S. D.; VASCONCELOS, M. C.; BLANK, A. F. Genetic variability in Jatropha curcas L. from diallel crossing. Genetics and Molecular Research, Ribeirão Preto, v. 16, n. 2, p. 1-13, 2017. DOI: http://dx.doi.org/10.4238/gmr16029651

ROSADO, T. B.; LAVIOLA, B. G.; FARIA, D. A.; PAPPAS, M. R.; BHERING, L. L.; QUIRINO, B.; GRATTAPLAGLIA, D. Molecular markers reveal limited genetic diversity in a large germplasm collection of the biofuel crop Jatropha curcas L. in Brazil. Crop
Science, Madison, v. 50, n. 6, p. 2372-2382, 2010. DOI: https://dx.doi.org/10.2135/cropsci2010.02.0112

SANTOS, C. A. F.; DRUMOND, M. A.; RODRIGUES, M. A.; EVANGELISTA, M. R. V. Genetic similarity of Jatropha curcas L. accessions based on AFLP markers. Crop Breeding and Applied Biotechnology, Viçosa, v. 10, n. 4, p. 364-369, 2010. DOI: http://dx.doi.org/10.1590/S1984-70332010000400012

SOKAL, R. R.; ROHLF, F. J. The comparison of dendrograms by objeticve methods. Taxon, Berlin, v. 11, n. 1, p. $30-40,1962$. 